\title{
¿EXISTE RELACIÓN ENTRE EL CRECIMIENTO Y LA TOXICIDAD EN ALEXANDRIUM CATENELLA (DINOFLAGELLATA)?
}

\author{
DOES A RELATIONSHIP EXIST BETWEEN THE GROWTH AND TOXICITY \\ OF ALEXANDRIUM CATENELLA (DINOFLAGELLATA)?
}

Cristián Garrido \& Máximo Frangópulos ${ }^{2}$

Los dinoflagelados son organismos unicelulares que dominan en los ambientes marinos tropicales, subtropicales y también pueden destacarse dentro de los grupos fitoplanctónicos predominantes del verano y otoño de zonas temperadas y boreales. En ocasiones, pueden generar florecimientos de carácter nocivo (FAN o mareas rojas) (Levinton, 2009). Por lo que en el último tiempo el interés por estudiar organismos causantes de FAN ha incrementado a nivel mundial, ya sean a través de estudios ecológicos in situ o experimentos de cultivo y autoecología en laboratorio.

Dentro de los principales factores ambientales que controlan el desarrollo de las poblaciones de dinoflagelados, los nutrientes juegan un rol clave, sobretodo en la generación de floraciones tóxicas que pueden ocurrir en diferentes tipos de hábitats, desde ambientes oligotróficos a hiper-nutrificados. (GEOHAB 2005).

Además, los nutrientes afectan la relación entre el crecimiento celular y la producción de toxinas, en algunos casos induciéndola y en otros, disminuyéndola (Cembella \& John, 2006). Asimismo, distintas especies de dinoflagelados pueden "preferir" distintos nutrientes o diferentes formas del mismo nutriente, por lo mismo, no siempre ambientes eutrofizados llevarán a la formación de floraciones (Anderson et al. 2002), pero sí se ha demostrado que los micronutrientes y macronutrientes tiene una profunda influencia en la expresión de las toxinas (Graneli \& Flynn, 2006).

El presente estudio consistió en analizar si existe alguna relación entre el crecimiento y la producción de toxinas en el dinoflagelado Alexandrium catenella, fuente primaria del Veneno Paralizante de los Mariscos (VPM) en el sur de Chile, bajo diferentes proporciones de nitratos y fosfatos.

Para ello se cultivó la cepa AY2 de A. catenella aislada en la región de Aysén en medio L1 (Guillard \& Hargraves, 1993) durante 30 días, bajo las siguientes condiciones de laboratorio: ciclo luz-oscuridad 14h:10h, salinidad de 30 PSU, tem-

1 Instituto de Fomento Pesquero, base zonal Punta Arenas, Enrique Abello 0552 Punta Arenas, Chile. cristián.garrido@ifop.cl

2 Centro Regional de Estudios del Cuaternario de Fuego-Patagonia y Antártica (Fundación CEQUA); Av. 21 de Mayo 1690, Casilla 737, Punta Arenas, Chile. 
peratura de $15^{\circ} \mathrm{C}$ y intensidad de luz promedio de $24 \mu \mathrm{Em}^{-2} \mathrm{~s}^{-1}$. Simultáneamente, se prepararon las siguientes proporciones nitrato/fosfato: Proporción de nitrógeno alto $(\mathrm{N}: \mathrm{P}=24: 1)$, balanceada $(\mathrm{N}: \mathrm{P}=$ $12: 1)$ y limitante (N:P = 3:1), el fósforo se consideró limitante en los 3 escenarios.

Para mayor detalle acerca de las metodologías de cultivo, ver Garrido et al. 2012 (este número).

En N:P = 24:1 se encontró una relación directamente proporcional y significativa entre ambas variables $(R=0,94, p<0,05)$ esto es, a medida que aumentaba la tasa de crecimiento, la concentración de toxinas se comportaba de la misma manera. El valor de coeficiente de determinación para esta proporción $\left(\mathrm{R}^{2}=0,89\right)$ nos indicó que la variabilidad de las toxinas puede ser explicada por los cambios en la tasa de crecimiento (Fig. 1a).

Con un $\mathrm{R}=0.83$ se presentó una relación directamente proporcional entre la tasa de crecimiento y la concentración de toxinas en N:P = 12:1 (Fig. 1b). Esto se explica porque al aumentar la tasa de crecimiento también lo hicieron las toxinas y, como en el caso anterior, nuevamente la variabilidad en las toxinas para esta concentración estuvo relacionada directamente con los cambios en la tasa de crecimiento. $\left(\mathrm{R}^{2}=0.70 ; \mathrm{p}<0,05\right)$.

El bajo valor observado para el coeficiente de correlación $(\mathrm{R}=0,09)$ en $\mathrm{N}: \mathrm{P}=3: 1$, indicó que estas dos variables no se relacionaron entre sí (Fig. 1c), luego, ambas aumentan o disminuyen de manera significativa $(p<0,05)$ de modo independiente una de la otra, aunque bajo este escenario no se observó ninguna relación lineal $\left(\mathrm{R}^{2}=0,01\right)$.

Las relaciones entre tasas de crecimiento y toxicidades en algas nocivas han sido estudiadas exhaustivamente por diversos autores (Taroncher et al. 1999, Maestrini et al. 1999, Guisande et al. 2002, Murata et al. 2006, Wang et al. 2006). De ellos es importante destacar lo observado por Murata et al. (2006), quienes encontraron que el contenido total tóxico de Alexandrium tamarense mostraba una relación lineal positiva con el crecimiento, bajo diferentes proporciones de N:P, lo cual coincide con lo observado para este estudio, en dos de las tres proporciones utilizadas. El mismo autor también menciona que de existir en el medio un abastecimiento continuo y alto de Nitrógeno y Fósforo, se esperaría que las células presenten mayores tasas de crecimiento y acumulen más toxinas.
No obstante lo anterior, existen estudios realizados con otras especies de Alexadrium que señalan resultados opuestos; por ejemplo Guisande et al. (2002) encontraron que una mayor producción de toxinas en Alexandrium minutum no estuvo
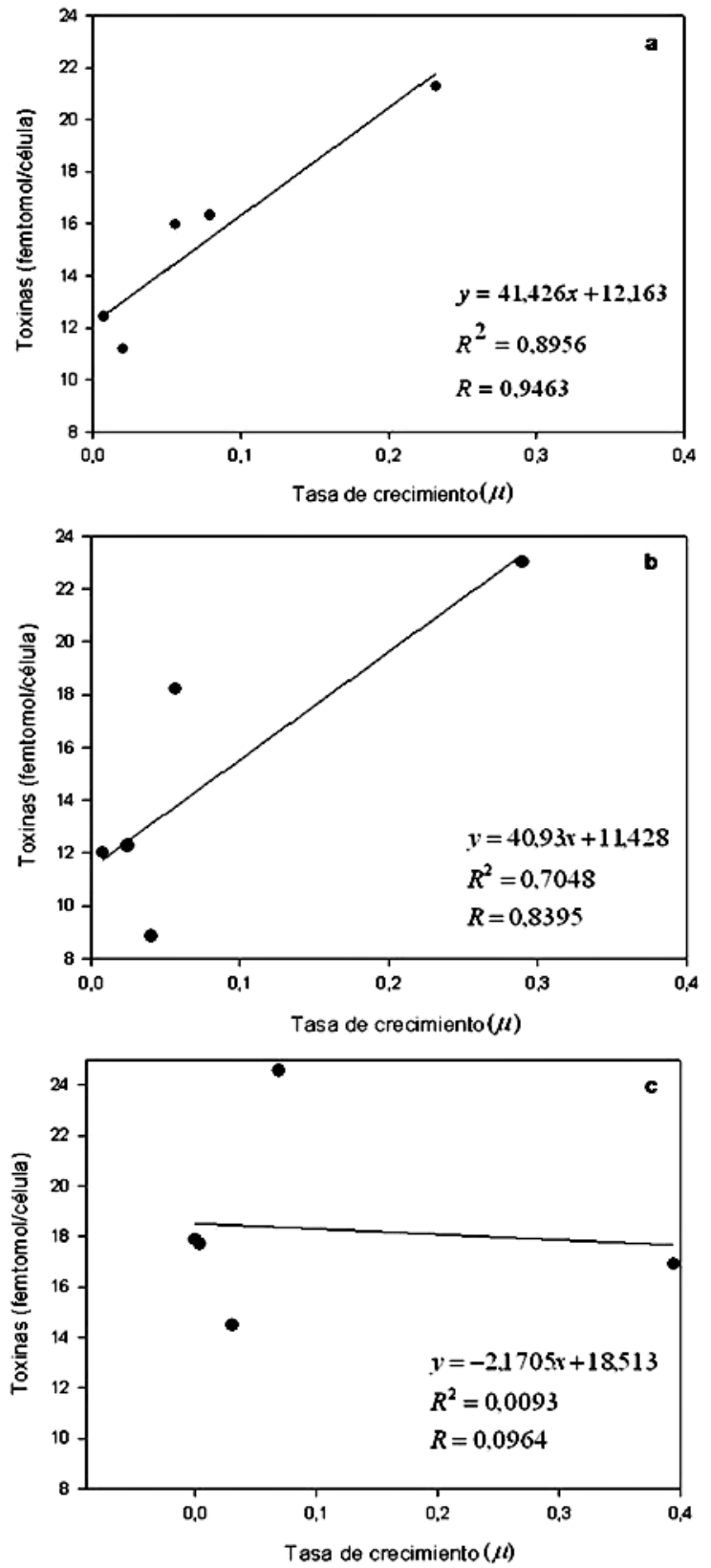

Fig. 1. Relación entre la toxicidad total y tasas de crecimiento en la proporción de nitrógeno alto $\mathrm{N}: \mathrm{P}=24: 1$ (a), balanceada $\mathrm{N}: \mathrm{P}=12: 1$ (b) y limitante $\mathrm{N}: \mathrm{P}=3: 1$ (c) 
asociada a su crecimiento, sino que se relacionaba con la disminución en las concentraciones de fósforo en el medio. Estos resultados ponen en evidencia las variaciones existentes en especies del mismo género, en especial en el caso de Alexandrium.

En la proporción de nitrógeno limitante, Alexandrium catenella no mostró una relación de dependencia entre las toxinas y el crecimiento, probablemente a consecuencia de las bajas proporciones utilizadas. Es decir fue incapaz de estimular suficientemente la producción de aminoácidos como la arginina, necesaria para la síntesis de biotoxinas (Shimizu et al. 1985). En un escenario en el cual el proceso de inhibición de la arginina hubiera sido total, se esperaría que la relación fuese inversa.

Taroncher et al. (1999) al respecto señalaron que en el caso de Alexandrium fundyense estas relaciones, ya sean inversas o directas entre el crecimiento y la toxicidad, se deben a las condiciones de limitación de nutrientes, situación que conduce a variaciones del ciclo celular en el metabolismo interno de los dinoflagelados.

De esta manera el establecimiento de relaciones entre el crecimiento y toxicidad en microalgas tóxicas como Alexandrium catenella bajo distintas proporciones de nutrientes en condiciones de laboratorio, se convierte en una herramienta que permite evaluar su fisiología y comportamiento en la naturaleza y cuál sería el impacto que tendría su toxicidad en base a sus tasas de crecimiento.

\section{AGRADECIMIENTOS}

Se agradece al Instituto de Fomento Pesquero (IFOP) Base Zonal Punta Arenas, por facilitar las instalaciones para poder realizar el presente estudio, especialmente a Claudia Zamora por los análisis de toxinas realizados por HPLC. Al Dr. Daniel Varela, de la Universidad de Los Lagos, encargado del cepario del centro i-mar, quién cedió gentilmente la cepa AY2 de Alexandrium catenella, con la cual se llevó a cabo este trabajo.

El presente estudio fue financiado en su totalidad por el proyecto FONDECYT regular $\mathrm{N}^{\circ} 1080548$.

\section{LITERATURA CITADA}

Anderson, D., P. Glibert \& J. Burkholder 2002. Harmful algal blooms and Eutrophication: Nutrient Sources, composition and consequences. Estuaries, 25(4b): 704-726.

Cembella, A \& U. John 2006. Molecular Physiology of Toxin Production and Growth Regulation in Harmfull Algae In: Granéli, E \& Turner J. (Eds.) Ecology of Harmful Algae, Ecological Studies Vol. 189, Springer, Berlin, Heidelberg pp. 215-226. ISBN: 3-540-32209.

Garrido, C., M. Frangópulos \& D. Varela 2012. Efecto de diferentes proporciones de nitrógeno/fósforo en el crecimiento y toxicidad de Alexadrium catenella (Dinoflagellata). Anales del Instituto de la Patagonia, 40(2): 115-125.

GEOHAB, 2005. Global ecology and oceanography of harmful algal blooms. In: Pitcher, G., Moita, T., Trainer, V., Kudela, R., Figueiras, F., Probyn, T. (Eds.), GEOHAB Core Research Project: HABs in Upwelling Systems. SCOR and IOC,Paris and Baltimore. $82 \mathrm{pp}$.

Guillard, R. \& P. Hargraves 1993. Stichochrysis immobilis is a diatom, not a chrysophyte. Phycologia 32: 234-236.

Guisande, C., M. Frangópulos, I. Maneiro, AR, Vergara \& I, Riveiro. 2002. Ecological advantages of toxin production by the dinoflagellate Alexandrium minutum under phosphorus limitation. Marine Ecology Progress Series. 225: 169-176.

Levinton, J. 2009. Marine Biology, Function, Biodiversity, Ecology. Third Edition,pp 640. Oxford University Press, New York USA, ISBN: 978-0-19-532694-9.

Maestrini, S., C. Bechemin, D. Grzebyk \& C, Hummert. 1999. Phosphorus limitation might promote more toxin content in the marine invader dinoflagellate Alexandrium minutum. Biology Ecología, 47 (1): 7-11.

Murata, Ai., S. Chee, Y. Leong, Y. Nagashima \& S. Taguchi. 2006.Nitrogen:Phosphorus supply ratio may control the protein and total toxin of dinoflagellate Alexandrium tamarense. Toxicon, 48: 683-689. 
Shimizu, Y., S. Gupta, M. Norte, A. Hori, A. Genenah. \& M. Kobayashi 1985. Biosynthesis of paralytic shellfish toxins. In: Toxic Dinoflagellates (Anderson, D.M., White, A.W. \& Baden, D.G., editors), Elsevier Press, New York. 271-274.

Taroncher, G., D. Kulis \& D.M, Anderson 1999. Coupling of Saxitoxin Biosynthesis to the G1 Phase of the Cell Cycle in the Dinofagellate Alexandrium fundyense: Temperature and Nutrient Effects. Nat. Toxins. 7: 207-219.
Wang, D.Z., S. Zhang, H. Gu, L. Chan. \& H. Hong 2006. Paralytic shellfish toxin profiles and toxin variability of the genus Alexandrium (Dinophyceae) isolated from the Southeast China Sea. Toxicon, 48: 138-151. 